\title{
Synthesis and Antimicrobial Evaluation of 3-Hydrazino-Naphthoquinones as Analogs of Lapachol
}

\author{
Claudia G. T. Oliveira ${ }^{a, c}$, Frederico F. Miranda ${ }^{a}$, Vitor F. Ferreira ${ }^{a *}$, Cícero C. Freitas $^{b}$, \\ Renata F. Rabello ${ }^{b}$, Jupira M. Carballido ${ }^{d}$ and Luiz C. D. Corrêa ${ }^{b}$ \\ ${ }^{a}$ Universidade Federal Fluminense, Instituto de Química, Departamento de Química Orgânica, CEG, \\ Campus do Valonguinho, 24210-150, Niterói - RJ, Brazil \\ ${ }^{\mathrm{b}}$ Universidade Federal Fluminense, Instituto de Biologia, CEG, Campus do Valonguinho, 24210-150, Niterói - RJ, Brazil \\ ${ }^{\mathrm{c}}$ Núcleo de Pesquisa de Produtos Naturais, Universidade Federal do Rio de Janeiro, 21941-540, \\ Rio de Janeiro - RJ, Brazil \\ ${ }^{\mathrm{d}}$ Universidade Federal Fluminense, Hospital Universitário Antônio Pedro, 24030-210, Niterói - RJ, Brazil
}

\begin{abstract}
Vários derivados de 1,4-naftoquinonas contendo um grupo hidrazino como cadeia lateral foram sintetizados a partir do 3-diazo-naftaleno-1,2,4-triona e foram avaliados como potenciais agentes antimicrobianos. Os derivados naftoquinônicos 2-[N'-(1-acetil-2-oxo-propilideno)-hidrazino]-3hidroxi-[1,4]naftoquinona, 2-[(3-hidroxi-1,4-dioxo-1,4-diidro-naftaleno-2-il)-hidrazono]-3-oxobutirato de etila, 2-[(3-hidroxi-1,4-dioxo-1,4-diidro-naftaleno-2-il)-hidrazono]-3-oxo-butirato de $t$-butila, 3-hidroxi-2-[(di-O-isopropilideno-malonato)-hidrazino]-1,4-naftoquinona e 2-[(3-hidroxi1,4-dioxo-1,4-diidro-naftaleno-2-il)-hidrazono]-malonato de etila mostraram maior atividade antibacteriana, ao nível de teste preliminar em disco, que o lapachol (1), uma 1,4-naftoquinona muito conhecida pelas suas variadas atividades biológicas. Estudo sobre a concentração mínima inibitória (MIC) para o Staphylococcus aureus mostrou que 2-[(3-hidroxi-1,4-dioxo-1,4-diidronaftaleno-2-il)-hidrazono]-malonato de etila tem uma atividade duas vezes maior que 1. Da mesma forma, o estudo da densidade ótica em cultura de $S$. aureus com esta substância mostrou uma atividade similar à da vancomicina na concentração de 2 XMIC.
\end{abstract}

Several 1,4-naphthoquinone derivatives having a hydrazino side chain were synthesized from 3-diazo-naphthalene-1,2,4-trione and tested as potential antimicrobial agents. These naphthoquinone derivatives 2-[N'-(1-acetyl-2-oxo-propylidene)-hydrazino]-3-hydroxy[1,4]naphthoquinone, ethyl 2-[(3-hydroxy-1,4-dioxo-1,4-dihydro-naphthalen-2-yl)-hydrazono]3-oxo-butyrate, $t$-butyl 2-[(3-hydroxy-1,4-dioxo-1,4-dihydro-naphthalen-2-yl)-hydrazono]-3-oxobutyrate, 3-hydroxy-2-[(di-O-isopropylidene-malonate)-hydrazino]-1,4-naphthoquinone, and diethyl 2-[(3-hydroxy-1,4-dioxo-1,4-dihydro-naphthalen-2-yl)-hydrazono]-malonate showed greater antibacterial activity at the level of the preliminary susceptibility testing in disk than lapachol (1), a well known 1,4-naphthoquinone which has several biological activities. Studies on the minimal inhibitory concentration (MIC) for Staphylococcus aureus showed that diethyl 2-[(3hydroxy-1,4-dioxo-1,4-dihydro-naphthalen-2-yl)-hydrazono]-malonate has an activity twofold greater than 1. On the other hand, optical density measurement for $S$. aureus indicated that this compound has similar activity compared with vancomycin at 2 xMIC.

Keywords: naphthoquinone, hydrazino-naphthoquinone, antimicrobial activity, lapachol

\section{Introduction}

Quinones have been the subject of much interest for a number of years due to their various biological activities. In 1946 Wendel $^{1}$ showed that certain 2-hydroxy-3-alkyl-

* e-mail: cegvito@vm.uff.br naphthoquinones inhibited the growth of Plasmodium. Further studies proved that the toxicity of naphthoquinones to Plasmodium $s p$. is due to interaction with the mitochondrial respiratory chain ${ }^{2}$. This observation led Fieser and collaborators to start an extensive search for new quinones ${ }^{3}$ aiming to discover new drugs for malaria chemotherapy. These resulted in the discovery of 3-(8- 
cyclohexyl-octyl-2-hydroxy-1,4-naphthoquinone (menoctone), a potent inhibitor of NADH and succinatecytochrome reductases of Plasmodium lophurae ${ }^{4}$. The antibacterial and antiprotozoan activities of 2-hydroxy-3alkyl-1,4-naphthoquinones have been summarized by several authors ${ }^{5,6}$. Besides these biological activities, various other quinones posses activities against several types of cancer cells ${ }^{7}$ (i.e. mitomycins, anthracyclines, etc.), virus $^{8,9}$ and fungi ${ }^{10}$.

Among the naphthoquinones, lapachol (1) and many heterocyclic derivatives have been investigated during the past years, mainly due to their antibacterial ${ }^{11}$, antifungal ${ }^{12}$ and anticancer ${ }^{13}$ activities. More recently, $\beta$-lapachone, an isomer of lapachol, was intensely investigated for clinical use in cancer chemotherapy ${ }^{14}$.

It is well known that there is a relationship between the side chain attached to 2-hydroxy-1,4-naphthoquinone and its toxic effects on several microorganisms ${ }^{15,16}$. Fieser and Richardson showed that, as the isoalkyl side chain of hydrolapachol is lengthened by the insertion of more methylene groups the activity against $P$. lophurae in the duck increased, reached a maximum with a C9-side chain, and then fell off ${ }^{17}$. Stern et al. found that the hydrogen peroxide formation of several 1,4-naphthoquinone derivatives on blood cell metabolism decreased with the increasing alkyl chain length ${ }^{18}$. As the alkyl side chain is an important factor for the activity of 2-hydroxy-1,4naphthoquinone, it seems appropriate to study the antimicrobial activity of naphthoquinone derivatives having new side chains linked to the quinone moiety.
The present work reports the synthesis and antibacterial activities of 3-hydroxy-2-hydrazino-1,4-naphthoquinone derivatives $\mathbf{2}$ (Figure 1) compared with $\mathbf{1}$ and other clinical antibiotics.<smiles>CC(C)=CCC1=C(O)C(=O)c2ccccc2C1=O</smiles><smiles>[R]C(=O)C(=NNC1=C(O)C(=O)c2ccccc2C1=O)C([R2])=O</smiles>

2a, $\mathrm{R}^{1}=\mathrm{R}^{2}=\mathrm{Me}$

2b, R1=Me, R2=OEt

2c, $\mathrm{R}^{1}=\mathrm{Me}, \mathrm{R} 2=\mathrm{O} t-\mathrm{Bu}$

2d, $\mathrm{R}^{1}=\mathrm{R}^{2}=\mathrm{OCMe}_{2} \mathrm{O}$

2e, $\mathrm{R}^{1}=\mathrm{R}^{2}=\mathrm{OEt}$

Figure 1. Lapachol (1) and hydrazino-1,4-naphthoquinone derivatives 2.

\section{Results and Discussion}

The synthesis of 3-hydroxy-2-hydrazino-1,4naphthoquinone derivatives $\mathbf{2 a - e}$ was performed by a modified process previously reported (Scheme 1$)^{19}$. Our synthesis of 2a-e started with lawsone 3 (3-hydroxy-1,4naphthoquinone) which was converted into nitroso-quinone $\mathbf{4}$ by nitrosation. Compound $\mathbf{4}$ was reduced with sodium dithionate in aqueous medium giving a moderate yield of 2-amino-3-hydroxy-1,4-naphthoquinone $\mathbf{5}^{20}$. Reduction of 4 using sodium borohydride in methanol produced 5 in similar yield. Diazotisation of the latter compound was<smiles>O=C1C=C(O)C(=O)c2ccccc21</smiles><smiles>CC(C)(C)O[C@H]1C(=O)C(N)=C(O)C(=O)c2ccccc21</smiles>

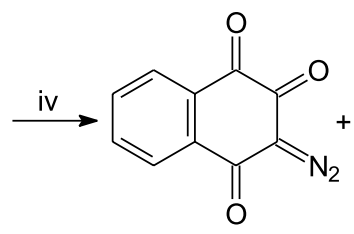

6, $68 \%$

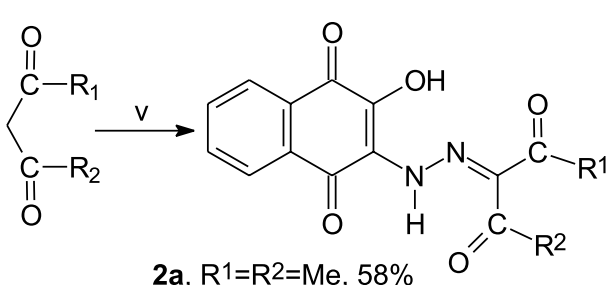

2b, $\mathrm{R}^{1}=\mathrm{Me}, \mathrm{R} 2=\mathrm{OEt}, 55 \%$

2c, $\mathrm{R}^{1}=\mathrm{Me}, \mathrm{R}^{2}=\mathrm{O} t-\mathrm{Bu}, 68 \%$

2d, $\mathrm{R}^{1}=\mathrm{R}^{2}=\mathrm{OCMe}_{2} \mathrm{O}, 58 \%$

2e, $\mathrm{R}^{1}=\mathrm{R}^{2}=\mathrm{OEt}, 55 \%$

i) $\mathrm{NaNO}_{2}, \mathrm{HCl}$ 0-5 OC; ii) $\mathrm{NaBH}_{4} / \mathrm{MeOH}$ or iii) $\mathrm{Na}_{2} \mathrm{~S}_{2} \mathrm{O}_{4} / \mathrm{EtOH}$;

iv) $\mathrm{NaNO}_{2} / \mathrm{HCl}$; v) $\mathrm{K}_{2} \mathrm{CO}_{3}$ /acetone

Scheme 1. Synthetic route used for preparing hydrazino-naphthoquinone derivatives 2a-e. 
effected using sodium nitrite in hydrochloric acid leading to 3-diazo-naphthalene-1,2,4-trione (6). Addition of 1,3dicarbonyl enolates to the diazo function of $\mathbf{6}$ gave the hydrazino-1,4-naphthoquinones 2a-e. These compounds were synthesized from lawsone $\mathbf{3}$ in 4 steps with overall yields varying from 16 to $20 \%$. Each step is operationally convenient and reproducible and the structures of 2a-e were supported by NMR data based on HMBC and HMQC experiments.

Table 1 reports the inhibition zones $(\mathrm{mm})$ of $\mathbf{2 a}, \mathbf{2 b}$, 2e, 1 and DMSO (solvent) determined for three species of Gram-positive and six Gram-negative bacteria, S. aureus, Staphylococcus epidermidis and the nonfermentable Gramnegative bacillus showed inhibition zones ranging from 9 to $24 \mathrm{~mm}$, where significant response requires $\geq 12 \mathrm{~mm}$. Other Gram-negatives and Enterococcus faecalis had no significant result (halo $<12 \mathrm{~mm}$ ). Therefore, our derivatives were active against four Gram-positive strains, but only against one Gram-negative strain. On the other hand, while 1 exhibited no positive response (halo $<12 \mathrm{~mm}$ ), DMSO was completely inactive (halo $=0$ ).

Table 1. Antimicrobial activity of $\mathbf{2 a}, \mathbf{2 b}, \mathbf{2 e}$ and $\mathbf{1}$ as determined by diffusion techniques.

\begin{tabular}{|c|c|c|c|c|c|c|}
\hline \multirow[b]{2}{*}{ Microorganism } & \multirow[b]{2}{*}{ Gram } & \multicolumn{5}{|c|}{ Inhibition zone (mm) } \\
\hline & & $\mathbf{2 a}$ & $2 \mathbf{b}$ & $2 e$ & $\begin{array}{l}\text { Lapachol } \\
\text { (1) }\end{array}$ & DMSO \\
\hline \multicolumn{7}{|l|}{ Nonfermentable } \\
\hline Gram-negative bacillus & & 12 & 12 & 16 & 10 & 0 \\
\hline Enterobacter $s p$ & - & 0 & 0 & 0 & 0 & 0 \\
\hline \multicolumn{7}{|l|}{ Enterococcus } \\
\hline faecalis & - & 0 & 0 & 9 & 0 & 0 \\
\hline Escherichia coli & + & 0 & 0 & 0 & 0 & 0 \\
\hline Klebsiella $s p$ & - & 0 & 0 & 0 & 10 & 0 \\
\hline Proteus mirabilis & - & 0 & 0 & 0 & 11 & 0 \\
\hline \multicolumn{7}{|l|}{ Pseudomonas } \\
\hline aeruginosa & - & 0 & 0 & 0 & 0 & 0 \\
\hline \multicolumn{7}{|l|}{ Staphylococcus } \\
\hline aureus 001 & + & 14 & 15 & 24 & 0 & 0 \\
\hline S. aureus 003 & + & 0 & 10 & 9 & 0 & 0 \\
\hline S. aureus 004 & + & 13 & 12 & 17 & 0 & 0 \\
\hline S. aureus ATCC $29.213^{\mathrm{a}}$ & + & 10 & 9 & 12 & 0 & 0 \\
\hline \multicolumn{7}{|l|}{ Staphylococcus } \\
\hline epidermidis & + & 11 & 15 & 16 & 0 & 0 \\
\hline
\end{tabular}

${ }^{a}$. aureus ATCC 29.213: oxacillin $=45 \mathrm{~mm}$ and vancomycin $=24 \mathrm{~mm}$.

The determinations of the minimal inhibitory concentrations (MIC) in clinical $S$. aureus strains are reported in Table 2. These results indicated that $\mathbf{2 e}$ has a greater activity than $\mathbf{1}$, but it is less active than oxacillin and vancomycin, which are currently used as clinical antibiotics.
Table 2. Minimal inhibitory concentration (MIC) for $\mathbf{2 e}$ and $\mathbf{1}$.

\begin{tabular}{lcc}
\hline Microorganism (Staphylococcus) & $\begin{array}{c}\mathbf{2 e} \\
\left(\mu \mathrm{g} \mathrm{mL}^{-1}\right)\end{array}$ & $\begin{array}{c}\text { Lapachol (1) } \\
\left(\mu \mathrm{g} \mathrm{mL}^{-1}\right)\end{array}$ \\
\hline S. aureus $001^{*}$ & 64 & 256 \\
S. aureus 002 & 128 & 256 \\
S. aureus 003 & 128 & 256 \\
S. aureus $004^{*}$ & 64 & 128 \\
S. aureus $005^{*}$ & 128 & 256 \\
S. aureus 006 & 128 & 256 \\
S. aureus $007^{*}$ & 128 & 256 \\
S. aureus $009^{*}$ & 128 & 256 \\
S. aureus 010 & 128 & 256 \\
S. aureus 012 & 128 & 256 \\
S. aureus 013 & 128 & 256 \\
S. aureus 015 & 128 & 256 \\
S. aureus $033^{*}$ & 128 & 256 \\
S. aureus $047^{*}$ & 128 & 256 \\
S. aureus $048^{*}$ & 128 & 256 \\
S. aureus ATCC 25.923 & 128 & 256 \\
S. aureus ATCC 29.213 & 128 & 128 \\
\hline
\end{tabular}

*MRSA - Methicillin-resistant S. aureus. MICs for S. aureus ATCC 29.213: oxacillin $=0.25 \mathrm{mg} \mathrm{mL}^{-1}$ and vancomycin $=2 \mathrm{mg} \mathrm{mL}^{-1}$.

Effects on the culture's optical density

The effects on the culture's optical density at $560 \mathrm{~nm}$ for $S$ aureus strains, were also studied. Cultures in the logarithimic growthphase were exposed to compound $2 \mathbf{e}$ and vancomycin, both at $2 x \mathrm{MIC}$, during a $6 \mathrm{~h}$ treatment, with measurements being made at each hour. As is shown in Figure 2, 2e and vancomycin exhibited similar responses, despite the lower MIC of vancomycin.

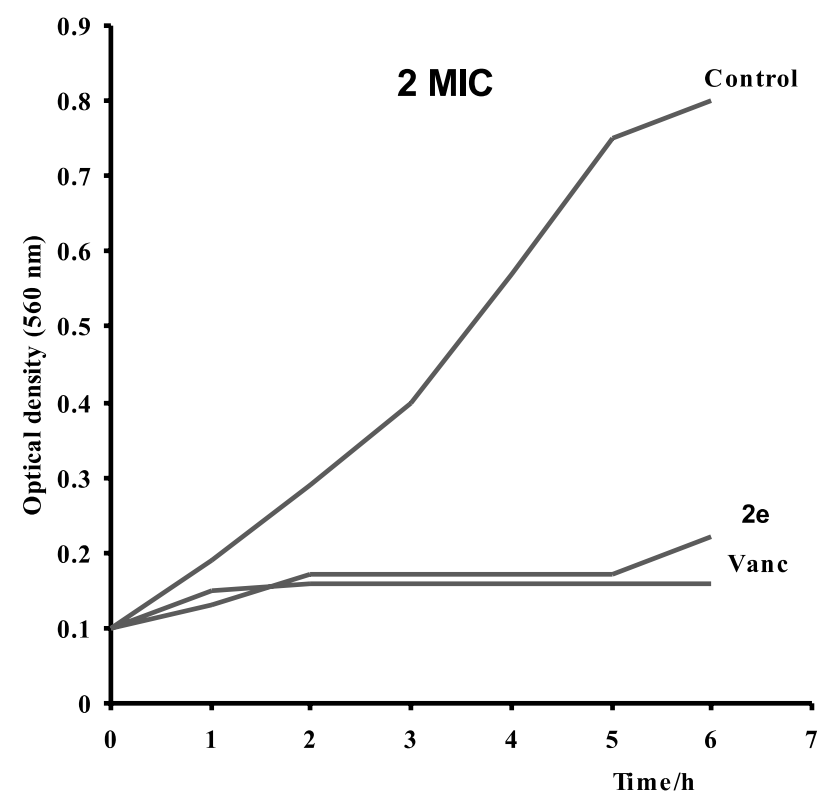

Figure 2. Effects of $2 \mathrm{e}$ and vancomycin on optical density of $S$. aureus cultures in exponential growth at $37^{\circ} \mathrm{C}$ ( MIC for $2 \mathrm{e}=64 \mu \mathrm{g} \mathrm{mL}^{-1}$ ). 


\section{Conclusion}

Our study revealed a new class of naphthoquinone derivatives with high antibacterial activity. The antibacterial activity of $\mathbf{2 a}, \mathbf{2 b}$ and $\mathbf{2 e}$ compared with $\mathbf{1}$ allow the conclusion that, at the level of the preliminary susceptibility testing in disk, they were active mainly against Grampositive bacteria. The minimal inhibitory concentration (MIC) determination on S. aureus for $\mathbf{2 e}$ showed an activity twofold greater than $\mathbf{1}$. However, $\mathbf{2 e}$ and $\mathbf{1}$ had MICs much greater than some clinical antibiotics. The optical density measurement for $S$. aureus indicated that $2 \mathrm{e}$ at $2 \times \mathrm{XMIC}$ had almost the same activity as vancomycin despite their different MICs.

\section{Experimental}

\section{General}

NMR and HRMS confirmed the structures of the substances. ${ }^{1} \mathrm{H}$ and ${ }^{13} \mathrm{C}$ NMR spectra were recorded with a Varian Unity Plus VXR spectrometer operating at 300 and $75 \mathrm{MHz}$ respectively, with tetramethylsilane as internal standard. Low resolution electron-impact mass spectra (12 eV) were obtained using a Hewlett Packard 5985 instrument and high resolution fast atom bombardment mass spectra (HRFABMS) were recorded in a 3-NBA matrix in the positive ion mode on a VG ZAB-E mass spectrometer. Infrared spectra were recorded on a Perkin-Elmer 783 spectrophotometer. Melting points were observed on a Reichert micro hotstage and are uncorrected. The solvents used were of analytical grade. Column chromatography was performed with silica gel 60 (Merck 70-230 mesh). Merck silica gel F254 (0.2 mm) was used for TLC plates, detection being carried out by spraying with a $25 \%$ aqueous solution of ammonium sulfate, followed by heating. Solvents were dried using the appropriate drying agents and then distilled directly before use ${ }^{21}$. Freshly purified samples were used for measurement of physical constants and spectral data. 2-hydroxy-1,4-naphthoquinone was purchased from Aldrich Chemical Co and used without purification.

\section{2-Hydroxy-3-nitroso-1,4-naphthoquinone (3)}

A mixture of lawsone $3(1 \mathrm{~g}, 5.75 \mathrm{mmol})$ in $\mathrm{HCl} 5 \%$ $(\mathrm{v} / \mathrm{v}, 7.5 \mathrm{~mL})$ under stirring was dissolved in dioxane $(20$ $\mathrm{mL}$ ). The solution was cooled externally with ice and solid sodium nitrite $(1.16 \mathrm{~g}, 16.8 \mathrm{mmol})$ was slowly added keeping the temperature below $5{ }^{\circ} \mathrm{C}$. The reaction was monitored by TLC until complete consumption of $\mathbf{3}$. The reaction was allowed to warm to room temperature and extracted with dichloromethane ( $3 \times 20 \mathrm{~mL})$. The combined organic phase was extracted with cold water $(3 \times 15 \mathrm{~mL})$ and dried over anhydrous $\mathrm{Na}_{2} \mathrm{SO}_{4}$ and concentrated under reduced pressure yielding $4(1.16 \mathrm{~g}, 90 \%)$ as a yellow crystalline solid; mp $169^{\circ} \mathrm{C}\left(\mathrm{CH}_{2} \mathrm{Cl}_{2}\right)$; IR $v_{\text {max }} / \mathrm{cm}^{-1} 3310$ $(\mathrm{OH}), 1690,1665(\mathrm{C}=\mathrm{O}), 1530(\mathrm{C}-\mathrm{N}=\mathrm{O})(\mathrm{KBr}) ;{ }^{1} \mathrm{H}$ NMR $\left(300 \mathrm{MHz}, \mathrm{CDCl}_{3}\right) \delta 8.25\left(1 \mathrm{H}, \mathrm{dd}, J 7.6\right.$ and $\left.1.1 \mathrm{~Hz}, \mathrm{H}_{5}\right)$, $8.20\left(1 \mathrm{H}, \mathrm{dd}, J 7.6\right.$ and $\left.1.1 \mathrm{~Hz}, \mathrm{H}_{8}\right), 7.93(1 \mathrm{H}, \mathrm{dt}, J 7.6$ and $\left.1.4 \mathrm{~Hz}, \mathrm{H}_{6}\right), 7.84\left(1 \mathrm{H}, \mathrm{dt}, J 7.6\right.$ and $\left.1.4 \mathrm{~Hz}, \mathrm{H}_{7}\right) ;{ }^{13} \mathrm{C} \mathrm{NMR}$ $\left(75 \mathrm{MHz}, \mathrm{CDCl}_{3}\right) \delta 173.1(\mathrm{C} 1), 159.2(\mathrm{C} 2), 136.0(\mathrm{C} 3)$, 183.0 (C4), 132.3 (C4a), 126.3 (C5), 135.0 (C6), 132.8 (C7), 126.1 (C8), 130.6 (C8a); MS m/z (relative abundance \%) $\mathrm{M}^{+} .203$ (20), 172 (72), 104 (100), 76 (48).

\section{2-Hydroxy-3-amino-1,4-naphthoquinone (5)}

A stirred solution of $4(1 \mathrm{~g}, 4.90 \mathrm{mmol})$ in ethanol (10 $\mathrm{mL}$ ) was warmed to $50^{\circ} \mathrm{C}$ then a freshly prepared solution of $\mathrm{Na}_{2} \mathrm{~S}_{2} \mathrm{O}_{4} 10 \%(\mathrm{w} / \mathrm{v}, 15 \mathrm{~mL})$ was added slowly in three portions of $5 \mathrm{~mL}$. The mixture changed from yellow to deep purple and after $15 \mathrm{~min}$ a solid started to form. The reaction was kept undisturbed at room temperature for 24 $\mathrm{h}$. The solid material was collected by vacuum filtration and air dried giving 5 (465 mg, 50\%) as a purple solid; mp $132{ }^{\circ} \mathrm{C}$ (EtOH) (lit. 130-140 $\left.{ }^{\circ} \mathrm{C}\right)^{20}$; IR $v_{\max } / \mathrm{cm}^{-1} 3480$ $(\mathrm{OH}), 3390\left(\mathrm{NH}_{2}\right), 1660,1650(\mathrm{C}=\mathrm{O})(\mathrm{KBr}) ;{ }^{1} \mathrm{H}$ NMR $(300$ $\left.\mathrm{MHz}, \mathrm{CDCl}_{3}\right) \delta 7.99\left(1 \mathrm{H}, \mathrm{dd}, J 6.9\right.$ and $\left.3.3 \mathrm{~Hz}, \mathrm{H}_{5}\right), 8.05$ $\left(1 \mathrm{H}, \mathrm{dd}, J 6.9\right.$ and $\left.3.3 \mathrm{~Hz}, \mathrm{H}_{8}\right), 7.62(1 \mathrm{H}, \mathrm{dt}, J 6.9$ and 2.4 $\left.\mathrm{Hz}, \mathrm{H}_{6}\right), 7.72\left(1 \mathrm{H}, \mathrm{dt}, J 6.9\right.$ and $\left.2.4 \mathrm{~Hz}, \mathrm{H}_{7}\right) ;{ }^{13} \mathrm{C}$ NMR $(75$ $\left.\mathrm{MHz}, \mathrm{CDCl}_{3}\right) \delta 177.0(\mathrm{C} 1), 135.9(\mathrm{C} 2), 133.2(\mathrm{C} 3), 181.4$ (C4), 130.3 (C4a), 125.1 (C5 ), 132.5 (C6), 133.8 (C7), 124.9 (C8), 131.0 (C8a).

\section{3-Diazo-naphthalene-1,2,4-trione (6)}

To a solution of 5 (200 mg, $1.05 \mathrm{mmol})$ in $\mathrm{HCl} 5 \%$ $(\mathrm{V} / \mathrm{V}, 4.0 \mathrm{~mL})$ and dioxane $(10 \mathrm{~mL})$ was slowly added solid sodium nitrite $(220 \mathrm{mg}, 3.19 \mathrm{mmol})$ at $0{ }^{\circ} \mathrm{C}$ during $1 \mathrm{~h}$. The mixture was stirred for an additional $30 \mathrm{~min}$ and extracted with dichloromethane $(2 \times 20 \mathrm{~mL})$. The combined organic phase was washed with an aqueous solution of $\mathrm{NaHCO}_{3} 5 \%$ ( $3 \times 5 \mathrm{~mL}$ ), dried over $\mathrm{Na}_{2} \mathrm{SO}_{4}$ and concentrated under reduced pressure producing a yellow solid which was crystallized from carbon tetrachloride yielding 6 (143 mg, $68 \%$ ); mp 121-122 ${ }^{\circ} \mathrm{C}\left(\mathrm{CCl}_{4}\right)$ (lit.123 $\left.{ }^{\circ} \mathrm{C}\right)^{22} ; \mathrm{IR} v_{\text {max }} / \mathrm{cm}^{-1} 2140(\mathrm{~N}=\mathrm{N}), 1690,1670,1630(\mathrm{C}=\mathrm{O})$ $(\mathrm{KBr}) ;{ }^{1} \mathrm{H}$ NMR $\left(300 \mathrm{MHz}, \mathrm{CDCl}_{3}\right) \delta 8.27(1 \mathrm{H}, \mathrm{dd}, J 6.0$ and $\left.2.4 \mathrm{~Hz}, \mathrm{H}_{5}\right), 8.25\left(1 \mathrm{H}\right.$, dd, $J 6.0$ and $\left.2.4 \mathrm{~Hz}, \mathrm{H}_{8}\right), 7.88$ 
$\left(1 \mathrm{H}, \mathrm{dt}, J 6.6\right.$ and $\left.1.5 \mathrm{~Hz}, \mathrm{H}_{7}\right), 7.81(1 \mathrm{H}, \mathrm{dt}, J 6.6$ and 1.5 $\left.\mathrm{Hz}, \mathrm{H}_{6}\right) ;{ }^{13} \mathrm{C} \mathrm{NMR}\left(75 \mathrm{MHz}, \mathrm{CDCl}_{3}\right) \delta 177.2$ (C1), 172.1 (C2), 148.6(C3), 176.5 (C4), 132.6(C4a), 126.2 (C5), 135.0 (C6), 134.5 (C7), 128.1 (C8), 133.1 (C8a); MS m/z (relative abundance \%) $\mathrm{M}^{+^{\circ}} 200$ (5), 172 (90), 104 (100), 76 (75).

\section{General procedure for preparing $2 a-e$}

A mixture of the appropriate dicarbonyl compound $(0.38 \mathrm{mmol})$, anhydrous $\mathrm{K}_{2} \mathrm{CO}_{3}(57 \mathrm{mg}, 0.4 \mathrm{mmol})$ in dry acetone $(15 \mathrm{~mL})$ was stirred for $15 \mathrm{~min}$ under a nitrogen atmosphere. To this mixture was added slowly through a syringe a solution of $\mathbf{6}(76.7 \mathrm{mg}, 0.38 \mathrm{mmol})$ in dry acetone $(5 \mathrm{~mL})$ during $15 \mathrm{~min}$. After stirring for $2 \mathrm{~h}$ the reaction was acidified with $5 \%(\mathrm{~V} / \mathrm{V}) \mathrm{HCl}(40 \mathrm{~mL})$, the solid material was collected by filtration and crystallized from ethanol.

2-[N'-(1-acetyl-2-oxo-propylidene)-hydrazino]-3-hydroxy[1,4]naphthoquinone $(2 a)$

$(66.7 \mathrm{mg}, 58 \%) ; \mathrm{mp} 195{ }^{\circ} \mathrm{C}\left(\mathrm{H}_{2} \mathrm{O}\right)\left(\text { lit } 206{ }^{\circ} \mathrm{C}\right)^{19}$; IR $v_{\text {max }} / \mathrm{cm}^{-1} 1675,1665,1640,1630(\mathrm{C}=\mathrm{O}), 1500(\mathrm{C}=\mathrm{N})$ $(\mathrm{KBr}) ;{ }^{1} \mathrm{H}$ NMR $\left(300 \mathrm{MHz}, \mathrm{CDCl}_{3}\right) \delta 8.16-8.12(2 \mathrm{H}, \mathrm{m}$, $\mathrm{H}_{5}$ and $\left.\mathrm{H}_{8}\right), 7.78-7.75\left(2 \mathrm{H}, \mathrm{m}, \mathrm{H}_{6}\right.$ and $\left.\mathrm{H}_{7}\right), 2.45\left(3 \mathrm{H}, \mathrm{s}, \mathrm{H}_{3}\right.$, ), $2.66\left(3 \mathrm{H}, \mathrm{s}, \mathrm{H}_{3}\right), 10.13\left(1 \mathrm{H}, \mathrm{s}\right.$, exchangeable with $\mathrm{D}_{2} \mathrm{O}$, $\mathrm{OH}), 14.50\left(1 \mathrm{H}\right.$, s, exchangeable with $\left.\mathrm{D}_{2} \mathrm{O}, \mathrm{NH}\right) ;{ }^{13} \mathrm{C} \mathrm{NMR}$ (75 MHz, DMSO) $\delta 179.3$ (C1), 146.1 (C2), 122.5 (C3), 180.5 (C4), 130.1 (C4a), 125.9 (C5), 134.6 (C6), 134.8 (C7), $125.9(\mathrm{C} 8), 130.2(\mathrm{C} 8 \mathrm{a}), 134.1(\mathrm{C}=\mathrm{N}), 196.5(\mathrm{MeC}=\mathrm{O}), 26.3$ $(\underline{\mathrm{MeC}}=\mathrm{O}), 196.7 \quad(\mathrm{MeC}=\mathrm{O}), 31.4(\underline{\mathrm{MeC}}=\mathrm{O}) ; \mathrm{MS} \mathrm{m} / \mathrm{z}$ (relative intensity, \%) $\mathrm{M}^{+} 300$ (35), 258 (100), 189 (65), 105 (25), 76 (20); HRFABMS found: 301.0832. Calcd for $[\mathrm{M}+\mathrm{H}]^{+} \mathrm{C}_{15} \mathrm{H}_{13} \mathrm{O}_{5} \mathrm{~N}_{2}: 301.0824$.

Ethyl 2-[(3-hydroxy-1,4-dioxo-1,4-dihydro-naphthalen-2yl)-hydrazono]-3-oxo-butyrate (2b)

(69.3 mg, 55\%); mp $163{ }^{\circ} \mathrm{C}\left(\mathrm{H}_{2} \mathrm{O}\right)\left(\text { lit. } 160-161{ }^{\circ} \mathrm{C}\right)^{19}$; IR $v_{\text {max }} / \mathrm{cm}^{-1} 1720,1675,1665,1645(\mathrm{C}=\mathrm{O}), 1500(\mathrm{C}=\mathrm{N})$ $(\mathrm{KBr}) ;{ }^{1} \mathrm{H}$ NMR $\left(300 \mathrm{MHz}, \mathrm{CDCl}_{3}\right) \delta 8.15-8.08(2 \mathrm{H}, \mathrm{m}$, $\mathrm{H}_{5}$ and $\left.\mathrm{H}_{8}\right), 7.75-7.72\left(2 \mathrm{H}, \mathrm{m}, \mathrm{H}_{6}\right.$ and $\left.\mathrm{H}_{7}\right), 2.69\left(3 \mathrm{H}, \mathrm{s}, \mathrm{H}_{3}\right)$, $4.34\left(2 \mathrm{H}, \mathrm{q}, J 7.2 \mathrm{~Hz}, \mathrm{H}_{3}\right), 1.40\left(3 \mathrm{H}, \mathrm{t}, J 7.2 \mathrm{~Hz}, \mathrm{H}_{4}\right), 14.00$ $\left(1 \mathrm{H}, \mathrm{s}\right.$, exchangeable with $\left.\mathrm{D}_{2} \mathrm{O}, \mathrm{NH}\right) ;{ }^{13} \mathrm{C} \mathrm{RMN}(75 \mathrm{MHz}$, $\left.\mathrm{CDCl}_{3}\right) \delta 179.1(\mathrm{C} 1), 145.7$ (C2), 122.3 (C3), $178.4(\mathrm{C} 4)$, 129.5 (C4a), 126.1 (C5), 134.0 (C6), $134.1\left(\mathrm{C}_{7}\right), 126.6$ (C8), $130.8(\mathrm{C} 8 \mathrm{a}), 124.9(\underline{\mathrm{C}}=\mathrm{N}), 196.9(\mathrm{Me} \underline{\mathrm{C}}=\mathrm{O}), 30.9(\underline{\mathrm{MeC}}=\mathrm{O})$, 162.2 ( $\underline{\mathrm{COOE}}), 61.6\left(\mathrm{OC}_{2} \mathrm{Me}\right), 14.1\left(\mathrm{OCH}_{2} \underline{\mathrm{Me}}\right) ; \mathrm{MS} \mathrm{m} / \mathrm{z}$ (relative intensity, \%) $\mathrm{M}^{+^{\circ}} 330$ (55), 288 (100), 188 (70), 104 (50), 76 (30); HRFABMS Found: 331.093. Calcd for $[\mathrm{M}+\mathrm{H}]^{+} \mathrm{C}_{16} \mathrm{H}_{15} \mathrm{O}_{6} \mathrm{~N}_{2}: 331.0930$.
t-Butyl 2-[(3-hydroxy-1,4-dioxo-1,4-dihydro-naphthalen2-yl)-hydrazono]-3-oxo-butyrate (2c)

(93.8 mg, 68\%); mp $162{ }^{\circ} \mathrm{C}$; IR $v_{\max } / \mathrm{cm}^{-1} 1740,1680$, $1690,1640(\mathrm{C}=\mathrm{O}), 1520(\mathrm{C}=\mathrm{N})(\mathrm{KBr}) ;{ }^{1} \mathrm{H} \mathrm{NMR}(300 \mathrm{MHz}$, $\left.\mathrm{CDCl}_{3}\right) \delta 8.15-8.08\left(2 \mathrm{H}, \mathrm{m}, \mathrm{H}_{5}\right.$ and $\left.\mathrm{H}_{8}\right), 7.75-7.72(2 \mathrm{H}, \mathrm{m}$, $\mathrm{H}_{6}$ and $\left.\mathrm{H}_{7}\right), 2.67\left(3 \mathrm{H}, \mathrm{s}, \mathrm{H}_{3}\right), 1.59\left(9 \mathrm{H}, \mathrm{s}, \mathrm{H}_{4}\right), 12.48(1 \mathrm{H}, \mathrm{s}$, exchangeable with $\left.\mathrm{D}_{2} \mathrm{O}, \mathrm{OH}\right), 14.20(1 \mathrm{H}, \mathrm{s}$, exchangeable with $\left.\mathrm{D}_{2} \mathrm{O}, \mathrm{NH}\right) ;{ }^{13} \mathrm{C} \mathrm{NMR}\left(75 \mathrm{MHz}, \mathrm{CDCl}_{3}\right) \delta 179.1$ (C1), 145.7 (C2), 122.5 (C3), 178.5 (C4), 129.5 (C4a), 126.1 (C5), 133.9 (C6), 134.1 (C7), 126.6 (C8), 130.8 (C8a), $123.0(\underline{\mathrm{C}}=\mathrm{N}), 197.1(\underline{\mathrm{MeC}}=\mathrm{O}), 31.1(\underline{\mathrm{MeC}}=\mathrm{O}), 161.4(\mathrm{t}-$ $\mathrm{BuOC}=\mathrm{O}), 82.9 \quad\left(\mathrm{Me}_{3} \underline{\mathrm{C}}\right), 28.0\left(\underline{\mathrm{Me}}_{3} \mathrm{C}\right) ; \mathrm{MS} \mathrm{m} / z$ (relative intensity, \%) 360 (10), 303 (45), 190 (55), 152 (60), 135 (98), 119 (100); HRFABMS Found: 359.1247. Calcd for $[\mathrm{M}+\mathrm{H}]^{+} \mathrm{C}_{18} \mathrm{H}_{16} \mathrm{O}_{6} \mathrm{~N}_{2}: 359.1243$.

5-[(3-Hydroxy-1,4-dioxo-1,4-dihydro-naphthalen-2-yl)hydrazono]-2,2-dimethyl[1,3]dioxane-4,6-dione (2d)

(66.7 mg, 58\%); mp $205^{\circ} \mathrm{C}$; IR $v_{\max } / \mathrm{cm}^{-1} 1740,1690$, 1670, $1650(\mathrm{C}=\mathrm{O}), 1540(\mathrm{C}=\mathrm{N})(\mathrm{KBr}) ;{ }^{1} \mathrm{H} \mathrm{NMR}(300 \mathrm{MHz}$, $\left.\mathrm{CDCl}_{3}\right) \delta 8.17-8.11\left(2 \mathrm{H}, \mathrm{m}, \mathrm{H}_{5}\right.$ and $\left.\mathrm{H}_{8}\right), 7.98-7.93(2 \mathrm{H}, \mathrm{m}$, $\mathrm{H}_{6}$ and $\left.\mathrm{H}_{7}\right), 1.86\left(3 \mathrm{H}, \mathrm{s}, \mathrm{H}_{4}\right) ;{ }^{13} \mathrm{C} \mathrm{NMR}(75 \mathrm{MHz}, \mathrm{DMSO})$ $\delta 180.6$ (C1), 148.3 (C2), 121.7 (C3), 178.7 (C4), 130.3 (C4a), 126.0 (C5), 134.1 (C6), 134.8 (C7), 126.1 (C8), 130.4 (C8a), $114.5(\underline{\mathrm{C}}=\mathrm{N}), 173.8(\underline{\mathrm{C}}=\mathrm{O}), 105.6\left(\mathrm{O} \underline{\mathrm{C}}(\mathrm{Me})_{2} \mathrm{O}\right), 27.1$ $\left(\mathrm{C}(\underline{\mathrm{Me}})_{2}\right)$; HRFABMS Found: 345.0722 . Calcd. for $[\mathrm{M}+\mathrm{H}]^{+}$ $\mathrm{C}_{16} \mathrm{H}_{13} \mathrm{O}_{7} \mathrm{~N}_{2}: 345.0722$.

Diethyl 2-[(3-hydroxy-1,4-dioxo-1,4-dihydro-naphthalen2-yl)-hydrazono]-malonate (2e)

(76 mg, 55\%); mp $184{ }^{\circ} \mathrm{C}$ (lit. $\left.186{ }^{\circ} \mathrm{C}\right)^{19}$; IR $v_{\max } / \mathrm{cm}^{-1}$ 1740, 1670, $1650(\mathrm{C}=\mathrm{O}), 1540(\mathrm{C}=\mathrm{N})(\mathrm{KBr}) ;{ }^{1} \mathrm{H}$ NMR (300 MHz, $\left.\mathrm{CDCl}_{3}\right) \delta 8.15-8.06\left(2 \mathrm{H}, \mathrm{m}, \mathrm{H}_{5}\right.$ and $\left.\mathrm{H}_{8}\right), 7.77-$ $7.70\left(2 \mathrm{H}, \mathrm{m}, \mathrm{H}_{6}\right.$ and $\left.\mathrm{H}_{7}\right), 4.46\left(2 \mathrm{H}, \mathrm{q}, J 7.2 \mathrm{~Hz}, \mathrm{H}_{3}\right), 4.34$ $\left(2 \mathrm{H}, \mathrm{q}, J 7.2 \mathrm{~Hz}, \mathrm{H}_{3}\right), 1.44\left(3 \mathrm{H}, \mathrm{t}, J 7.2 \mathrm{~Hz}, \mathrm{H}_{4}\right), 1.38(3 \mathrm{H}$, t, J 7.2 Hz, H $), 11.15(1 \mathrm{H}, \mathrm{s}, \mathrm{OH}), 12.86(1 \mathrm{H}, \mathrm{s}, \mathrm{NH}) ;{ }^{13} \mathrm{C}$ NMR $\left(75 \mathrm{MHz}, \mathrm{CDCl}_{3}\right) \delta 179.1$ (C1), 143.9 (C2), 120.4 (C3), 178.6 (C4), 130.6 (C4a), 125.9 (C5), 134.0 (C6), 133.7 (C7), 126.4 (C8), 129.3 (C8a), $122.2(\underline{C}=\mathrm{N}), 160.2$ $(\underline{\mathrm{C}}=\mathrm{O}), 61.6\left(\mathrm{OCH}_{2} \mathrm{Me}\right), 13.7\left(\mathrm{OCH}_{2} \underline{\mathrm{Me}}\right) ; \mathrm{MS} \mathrm{m} / \mathrm{z}$ (relative intensity, \%) M+ 360 (55), 268 (100), 104 (80), 76 (45); HRFABMS Found: 361.1040. Calcd for $[\mathrm{M}+\mathrm{H}]^{+}$ $\mathrm{C}_{17} \mathrm{H}_{17} \mathrm{O}_{7} \mathrm{~N}_{2}: 361.1035$.

\section{Microbial cultures growth conditions}

Tested microorganisms included the following Grampositive bacteria: Enterococcus faecalis, Staphylococcus 
aureus and Staphylococcus epidermidis, and for Gramnegative: Enterobacter, Escherichia coli, Klebsiella sp, nonfermentable bacillus, Proteus mirabilis and, Pseudomonas aeruginosa. All bacteria used in this study were isolated from patients at the University Hospital Antônio Pedro/UFF-RJ and grown (at $37{ }^{\circ} \mathrm{C}$ ) in medium with peptone, yeast extract, sodium chloride and, dibasicsodium phosphate. Lorian disks (7 $\mathrm{mm}$ diameter) were soaked in $5 \mathrm{mg} \mathrm{mL}^{-1}$ of substances (2a-e) as solutions in dimethylsulfoxide (DMSO). Disks were put on an exponentially growing plated culture with appropriate dilution to $1.0 \times 10^{7}$ colony forming unit $\left(\mathrm{CFU} \mathrm{mL} \mathrm{mL}^{-1}\right)$. The plates were then incubated for $24 \mathrm{~h}$ at $37^{\circ} \mathrm{C}$. The results were recorded by measuring the zones surrounding the disk. Control disks containing DMSO, ATCC 29.213 of S. aureus and the antibiotics oxacillin and vancomycin were used as controls in the assay. Significant results: halo $\geq 12 \mathrm{~mm}$.

\section{Minimal inhibitory concentration (MIC)}

The hydrazino-quinone $\mathbf{2 e}$ was tested by using the two fold serial dilution in agar. $1 \mathrm{~mL}$ of $\mathrm{DMSO} /$ compound solution was added to $19 \mathrm{~mL}$ of the medium at $55-60{ }^{\circ} \mathrm{C}$ in order to reach a final concentration from 512 to $1 \mu \mathrm{g} \mathrm{mL}^{-1}$. These solutions were plated and after medium solidification, a drop of $S$. aureus in the logarithmic phase

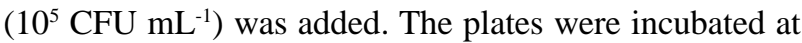
$37{ }^{\circ} \mathrm{C}$ for $24 \mathrm{~h}$, before being read. MIC was defined as the lowest compound concentration preventing visible bacterial growth. All strains were tested at least in duplicate in four separate experiments. MICs of the reference compound used in this study were similar to those found in other reports $^{23,24}$.

\section{Acknowledgments}

A fellowship granted to C.G.T.O. from CNPq (Brazil) is gratefully acknowledged. V.F.F. are grateful to $\mathrm{CNPq}$ for an individual research fellowship. This work was partially supported by CNPq (National Council of Research of Brazil), FAPERJ and Universidade Federal Fluminense.

\section{References}

1. Wendel, W. B. Fed. Proc. 1946, 5, 406.

2. (a) Ball, E. G.; Anfinsen, C. B.; Cooper, O. J. Biol. Chem. 1947, 168, 257; (b) Porter, T. H.; Folkers, K. Angew. Chem., Int. Ed. 1974, 13, 559.

3. Fieser, L. F.; Berligner, E.; Bondhus, F. J.; Chang, F. C.; Dauben, W. G.; Ettlinger, M. G.; Fawaz, G.; Fields, M.; Fieser, M.; Heildelberger, C.; Heymann, H.;
Seligman, A.M.; Vaughan, W. R.; Wilson, A. G.; Wilson, E.; Wu, M. -I.; Leffler, M. T.; Hamilin, K. E.; Hathaway, R. J.; Matson, E. J.; Moore, E. E.; Moore, M. B.; Rapala, R. T.; Zaugg, H. E. J. Am. Chem. Soc 1948, 70, 3151 and references cited therein.

4. (a) Fieser, L. F.; Schirmer, J. P.; Archer, S.; Lorez, R. R.; Pfaffenbach, P. I. J. Med. Chem. 1967, 10, 513 and references cited therein; (b) Fry, M.; Pudney, M. Biochem. Pharmacol. 1992, 43, 1545.

5. Hudson, A. T.; Randall, A. W.; Fry, M.; Ginger, C. D.; Hill, B.; Latter, V. S.; McHardy, N.; Willian, R. B. Parasitology 1985, 90, 45.

6. (a) Pinto, A. V.; Ferreira, V. F.; Capella, R. S.; Gilbert, B.; Pinto, M. C. R. F.; Silva, J. S. Trans. Roy. Soc. Trop. Med. Hyg. 1987, 81, 609; (b) Ferreira, V. F.; Pinto, A. V.; Filho, F. F.; Kamesiro, M. M.; Silva, I. S.; Santos, S. C.; Pinto, C. N.; Pinto, M. C. F. R.; Gilbert, B. Mem. Inst. Oswaldo Cruz 1992, 87, 345; (c) Leon, W.; Gonçalves, A. M.; Vasconcellos, M. E.; Do Campo, R.; Cruz, F. S.; Souza, V. Mol. Biochem. Parasitol. 1980, 1, 167; (d) Ribeiro-Rodrigues, R.; Santos, W. G.; Oliveira, A. B.; Snieckus, V.; Zani, C. L.; Romanha, A. J. Bioorg. Med. Chem. Lett. 1995, 5, 1509; (e) Boehm, P.; Cooper, K.; Hudson, A. T.; Elphick, J. P.; McHardy, N. J. Med. Chem. 1981, 24, 295; (f) McHardy, N.; Hudson, A. T.; Morgan, D. W. T.; Era, D. G.; Dolan, T. T. Rev. Vet. Sci. 1983, 35, 347.

7. (a) Kappus, H. Biochem. Pharmacol. 1986, 35, 1; (b) Tritton, T. R.; Yee, G. Science 1982, 217, 248; (c) Seguea-Aguilar, J.; Jonsson, K.; Tidefelt, U.; Paul, C. Leukemia Res. 1992, 16, 631; (d) Babich, H.; Stern, A. J. Appl. Toxicol. 1993, 13, 353; (e) Cheng, C.C.; Dong, Q.; Luo, D. F.; Liu, F.; Chen, A. Y.; Yu, C.; Savaraj, N.; Chou, T. C. J. Med. Chem. 1993, 36, 4108.

8. (a) Pinto, A. V.; Pinto, M. C. F. R.; Lagrota, M. H. C.; Wigg, M. D.; Aguiar, A. N. S. Rev. Microbiol. 1983, 14, 21; (b) Laatsch, H. Angew. Chem., Int. Ed. 1994, 33,422 .

9. Kanoshima, T.; Kozuka, M.; Koyama, J.; Okatami, T. J. Nat. Prod. 1989, 52, 987.

10. Monks, T. J.; Hanzlik, R. P.; Cohen, G. M.; Ross, D.; Grahen, D. G. Toxicol. Appl. Pharmacol. 1992, $112,2$.

11. (a) Kurylowicz, W. In Antibióticos: Uma Revisão Crítica, Kurylowicz, W., Ed., Guanabara Koogan, Brasil, Recife, 1981, p 226; (b) D'Albuquerque, I. L.; Maciel, M. C. N.; Schuler, R.P.; Araujo, M. C. M.; Maciel, G. M.; Cavalcanti, M. S. B.; Martins, D. G.; Lacerda, A. L. Rev. Inst. Antibiot. (Recife) 1971, 11, 21; (c) Cortes, M.; Katalinic, J.; Valderrama, J. An. Quím. 1983, 79c, 202. 
12. Guiraud, P.; Steiman, R.; Campos-Takaki, G. -M.; Seigle-Murandi, F.; De Buochberg, M. S. Planta Med. 1994, 60, 373.

13. (a) Rao, K. V.; McBride, T. J.; Oleson, J. J. Cancer Res. 1968, 28, 1952; (b) Lui, C.Y.; Ayeni, A. A.; Gyllenhall, C.; Groves, M. J. Drug. Dev. Ind. Pharm. 1985, 11, 1763; (c) Consolação, M. D.; Linardi, F.; Oliveira, M. M. D.; Sampaio, M. R. P. J. Med. Chem. 1975, 18, 1159; (d) Block, J. B.; Serpick, A. A.; Miller, W.; Wiernik, P. H. Cancer Chemother. Res. 1974, 4, 27; (e) Subramanian, S.; Ferreira, M. M. C.; Trsic, M. Struct. Chem. 1998, 9, 47.

14. (a) Pink, J. J.; Planchon, S. M.; Tagliarino, C.; Varnes, M. E.; Siegel, D.; Boothman, D. A. J. Biol. Chem. 2000, 275, 5416 and references cited therein; (b) Pink, J. J.; Wuerzberger-Davis, S.; Tagliarino, C.; Planchon, S. M.; Yang, X. H.; Froelich, C. J. Exp.Cell. Res. 2000, 255, 144; (c) Li, C. J.; Li, Y. -Z.; Pinto, A. V.; Pardee, A. B. Proc. Natl. Acad. Sci. (USA) 1999, 96, 13369; (d) Li, Y. Z.; Li, C. J.; Pinto, A. V.; Pardee, A. B. Mol. Med. 1999, 5, 232.
15. Mazunder, A.; Wang, S.; Neamati, N.; Nicklaus, M.; Sunder, S.; Chen, J.; Milne, G.; Rice, W.; Burke, R. T.; Pommer, Y. J. Med. Chem. 1996, 39, 2472.

16. Weaver, R. J.; Dickins, M.; Burke, M. D. Biochem. Pharmacol. 1993, 46, 1183.

17. Fieser, L. F.; Richardson, A. P. J. Am. Chem. Soc. 1948, 70, 3156.

18. Kruger-Zeitzer, E.; Sullivan, S. G.; Ster, A. J. Appl. Toxicol. 1990, 10, 129.

19. Reid, W.; Kahr, E. Liebigs Ann. Chem. 1969, 725, 228.

20. Podrebarac, G. E.; Cheng, C. C. J. Org. Chem. 1970, 35, 281.

21. Ferreira, V. F. Quím. Nova 1992, 15, 348.

22. Mosby, W. L.; Silva, M. L. J. Chem. Soc. 1964, 3990.

23. Bierbaun, G.; Fuchs, K.; Lenz, W.; Szekat, C.; Sahl, H. G. Eur. J. Clin. Microbiol. Infect. Dis. 1999, 18, 691.

24. Lorian, V.; Freitas, C. C. J. Infect. Dis. 1979, 139, 599.

Received: November 14, 2000 Published on the web: May 16, 2001 